\title{
EXTREME POINTS OF SUBORDINATION FAMILIES WITH UNIVALENT MAJORANTS
}

\author{
DAVID J. HALLENBECK
}

\begin{abstract}
Let $s(F)$ denote the set of functions subordinate to a univalent function $F$ in $\Delta$ the unit disc. Let $B_{0}$ denote the set of functions $\phi(z)$ analytic in $\Delta$ satisfying $|\phi(z)|<1$ and $\phi(0)=0$. We prove the following results: If $f=F \circ \phi$ is an extreme point of $s(F)$ and $F(\Delta)$ is a Jordan domain, then $\phi$ is an extreme point of $B_{0}$.
\end{abstract}

1. Introduction. Let $\Delta=\{z:|z|<1\}$ and let $\mathscr{Q}$ denote the set of functions analytic in $\Delta$. Let $B_{0}$ consist of the subset of $\mathscr{Q}$ consisting of all functions $\phi$ that satisfy the conditions $|\phi(z)|<1, \phi(0)=0$. Let $E B_{0}$ denote the extreme points of $B_{0}$. It is a classical fact [2, p. 125] that $\phi \in E B_{0}$ if and only if $\int_{0}^{2 \pi} \log \left(1-\left|\phi\left(e^{i \theta}\right)\right|\right) d \theta=-\infty$. Let $S$ denote the subset of $Q$ consisting of univalent functions $f$ so that $f(z)=z+\cdots$ in $\Delta$.

Let $F$ be in $Q$ and be univalent in $\Delta$. Let $s(F)$ denote the subset of $氏$ consisting of functions $f$ that are subordinate to $F$ in $\Delta$. This means that $f \in \mathbb{Q}, f(0)=F(0)$, and $f(\Delta) \subset F(\Delta)$. These conditions are equivalent to the existence of $\phi \in B_{0}$ so that $f=F \circ \phi$. Note that $s(F)=\left\{F \circ \phi: \phi \in B_{0}\right\}$.

Let $D$ denote $F(\Delta)$. Since $F \in H^{p}$ for $p<\frac{1}{2}\left[2\right.$, p. 50], if we define $f\left(e^{i \theta}\right)=$ $\lim _{r \rightarrow 1} f\left(r e^{i \theta}\right)$ then $f\left(e^{i \theta}\right)$ exists almost everywhere. Let $\lambda(\theta)$ denote the distance between $f\left(e^{i \theta}\right)$ and $\partial D$ where $\partial D$ denotes the boundary of $D$.

We let $H s(F)$ and $E H s(F)$ denote respectively the closed convex hull of $s(F)$ and the extreme points of the closed convex hull of $s(F)$. Since $s(F)$ is compact, $E H s(F) \subset s(F)$. Let $E s(F)$ denote the extreme points of $s(F)$. In [1], Yusuf Abu-Muhanna proved if $F^{\prime}$ is Nevanlinna and $D$ a Jordan domain subset to a half plane then $E H s(F) \subset\left\{F \circ \phi: \phi \in E B_{0}\right\}$. He conjectured that the inclusion holds for any univalent function $F$. We prove this inclusion only assuming $F$ is univalent and $D$ is a Jordan domain. Our arguments are similar to those of Abu-Muhanna [1].

2. Functions subordinate to a univalent function. We let $d(z, \Gamma)$ denote the distance between $z$ and a closed set $\Gamma, m(A)$ denote the Legesgue measure of $A$ and $\log ^{+} x=\max \{0, \log x\}$.

THEOREM 1. Let $F$ be in $Q$ and be univalent in $\Delta$. Whenever $g$ is in $s(F)$, let $\lambda(\theta)$ denote the distance between $g\left(e^{i \theta}\right)$ and $\partial D$ where $D=F(\Delta)$. Then when $g=F \circ \phi$,

(a) $\int_{0}^{2 \pi} \log ^{+} \lambda(\theta) d \theta$ is convergent and

Received by the editors June 9, 1983.

1980 Mathematics Subject Classification. Primary 30C80; Secondary 30C55.

Key words and phrases. Analytic functions, bounded function, extreme point, Jordan domains, Nevanlinna class, subordinations, univalent function.

(1984 American Mathematical Society $0002-9939 / 84 \$ 1.00+\$ .25$ per page 
(b) $\int_{0}^{2 \pi} \log \lambda(\theta) d \theta=-\infty$ implies $\int_{0}^{2 \pi} \log \left(1-\left|\phi\left(e^{i \theta}\right)\right|\right) d \theta=-\infty$.

If $F^{\prime}$ is Nevanlinna then

(c) $\int_{0}^{2 \pi} \log \left(1-\left|\phi\left(e^{i \theta}\right)\right|\right) d \theta=-\infty$ implies $\int_{0}^{2 \pi} \log \lambda(\theta) d \theta=-\infty$.

Proof. Since $F$ is univalent, it follows from [3, p. 22] that

$$
\frac{1}{4}\left(1-|z|^{2}\right)\left|F^{\prime}(z)\right| \leqslant d(F(z), \partial D) \leqslant\left(1-|z|^{2}\right)\left|F^{\prime}(z)\right|
$$

for all $z$ in $\Delta$. For $g$ in $s(F)$ we have $g=F \circ \phi$ where $\phi \in B_{0}$. If $\left|\phi\left(e^{i \theta}\right)\right|=1$ for some $\theta$ the univalence of $F$ gives $\lambda(\theta)=0$ and $\log ^{+} \lambda(\theta)=0$. When $g\left(e^{i \theta}\right)$ and $\phi\left(e^{i \theta}\right)$ exist, and $\left|\phi\left(e^{i \theta}\right)\right|<1$, we obtain from (1)

$$
\frac{1}{4}\left(1-\left|\phi\left(e^{i \theta}\right)\right|^{2}\right)\left|F^{\prime}\left(\phi\left(e^{i \theta}\right)\right)\right| \leqslant \lambda(\theta) \leqslant\left(1-\left|\phi\left(e^{i \theta}\right)\right|^{2}\right)\left|F^{\prime}\left(\phi\left(e^{i \theta}\right)\right)\right|,
$$

which implies $\lambda(\theta) \leqslant 2\left(1-\mid \phi\left(e^{i \theta}\right)\right)\left|F^{\prime}\left(\phi\left(e^{i \theta}\right)\right)\right|$. We may assume, without loss of generality, that $F \in S$ and so, it follows that

$$
\left|F^{\prime}(z)\right| \leqslant\left|\frac{F(z)}{z}\right| \frac{1+|z|}{1-|z|}
$$

for $z$ in $\Delta[3$, p. 21]. Hence,

$$
2\left(1-\left|\phi\left(e^{i \theta}\right)\right|\right)\left|F^{\prime}\left(\phi\left(e^{i \theta}\right)\right)\right| \leqslant 4\left|\frac{F\left(\phi\left(e^{i \theta}\right)\right)}{\phi\left(e^{i \theta}\right)}\right|
$$

and so

$$
0 \leqslant \log ^{+} \lambda(\theta) \leqslant \log ^{+} 4\left|\frac{F\left(\phi\left(e^{i \theta}\right)\right)}{\phi\left(e^{i \theta}\right)}\right| .
$$

It is known that $F(z) / z$ is in $H^{p}$ for each $p<\frac{1}{2}$ and so we have $F(\phi(z)) / \phi(z)$ in $H^{p}$ for each $p<\frac{1}{2}$. It follows that $\log ^{+}\left|F\left(\phi\left(e^{i \theta}\right)\right) / \phi\left(e^{i \theta}\right)\right|$ is integrable on $[0,2 \pi]$. It follows directly that (a) holds.

Now let $A=\left\{\theta: g\left(e^{i \theta}\right)\right.$ exists and $\left.\lambda(\theta)=0\right\}$. If $m(A)>0$ then it follows that $\int_{0}^{2 \pi} \log \lambda(\theta) d \theta=-\infty$. It also follows that $\int_{0}^{2 \pi} \log \left(1-\left|\phi\left(e^{i \theta}\right)\right|\right) d \theta=-\infty$ since $g\left(e^{i \theta}\right)$ on $\partial D$ implies that $\left|\phi\left(e^{i \theta}\right)\right|=1$, given that $F \in S$. Assume for the rest of the proof that $m(A)=0$. This implies (2) holds for almost all $\theta$. The fact that $1 \leqslant 1+\left|\phi\left(e^{i \theta}\right)\right| \leqslant 2$ applied to (2) gives

$$
\frac{1}{4}\left(1-\left|\phi\left(e^{i \theta}\right)\right|\right)\left|F^{\prime}\left(\phi\left(e^{i \theta}\right)\right)\right| \leqslant \lambda(\theta) \leqslant 2\left(1-\left|\phi\left(e^{i \theta}\right)\right|\right) \mid F^{\prime}\left(\phi\left(e^{i \theta}\right)\right)
$$

for almost all $\theta$. Since $F$ is in $S$, we have $\left[3\right.$, p. 21] $\left|F^{\prime}(z)\right| \geqslant(1-|z|) /(1+|z|)^{3}$ for $z$ in $\Delta$. Hence

$$
\left|F^{\prime}\left(\phi\left(e^{i \theta}\right)\right)\right| \geqslant \frac{1}{8}\left(1-\left|\phi\left(e^{i \theta}\right)\right|\right)
$$

and this fact, taken together with the left-hand side of (3) gives

$$
\lambda(\theta) \geqslant \frac{1}{4}\left(1-\left|\phi\left(e^{i \theta}\right)\right|\right)\left|F^{\prime}\left(\phi\left(e^{i \theta}\right)\right)\right| \geqslant \frac{1}{32}\left(1-\left|\phi\left(e^{i \theta}\right)\right|\right)^{2} .
$$

This implies

$$
-\log 32+2 \log \left(1-\left|\phi\left(e^{i \theta}\right)\right|\right) \leqslant \log \lambda(\theta) .
$$


Since $\log ^{+} \lambda(\theta) \in L^{1}[0,2 \pi]$ we have

$$
-\infty \leqslant \int_{0}^{2 \pi} \log \lambda(\theta) d \theta<M
$$

for some constant $M$. If $\int_{0}^{2 \pi} \log \lambda(\theta) d \theta=-\infty$ then (4) and (5) imply

$$
\int_{0}^{2 \pi} \log \left(1-\left|\phi\left(e^{i \theta}\right)\right|\right) d \theta=-\infty
$$

and (b) holds.

The proof of (c) is given in [1, p. 440].

3. Jordan domains. The following lemma appeared in [1, p. 444].

Lemma A. Let $D$ be a bounded Jordan domain. Let $g$ be a nonconstant bounded analytic function in $\Delta$. If $g\left(e^{i \theta}\right) \in \bar{D}$ for almost all $\theta$ then $g(\theta) \subset D$.

LEMMA 1. Let $D$ be an unbounded Jordan domain. Let $g$ be a nonconstant analytic function in $\Delta$ so that $g(\Delta) \cup D$ avoids an open set. If $g\left(e^{i \theta}\right) \in \bar{D}$ for almost all $\theta$ then $g(\Delta) \subset D$.

Proof. There exists a complex number $a$ and a $\delta>0$ so that $\{w:|w-a|<\delta\}$ $\cap\{g(\Delta) \cup D\}=\varnothing$. It follows that the function $h(z)=1 /(g(z)-a)$ is analytic and bounded for $z \in \Delta$. Also, $h\left(e^{i \theta}\right)=1 /\left(g\left(e^{i \theta}\right)-a\right)$ exists for almost all $\theta$ and $h\left(e^{i \theta}\right) \in \overline{f(D)}$ where $f(w)=1 /(w-a)$. It follows from Lemma A that $h(\Delta) \subset f(D)$ or $f(g(\Delta)) \subset f(D)$. Hence $g(\Delta) \subset D$.

THEOREM 2. Let $F$ be in $Q$ and univalent in $\Delta$ and suppose that $D=F(\Delta)$ is a Jordan domain. Let $\lambda(\theta)$ denote the distance between $f\left(e^{i \theta}\right)$ and $\partial D$ where $f \in s(F)$. If $f \in E s(F)$, then

$$
\int_{0}^{2 \pi} \log \frac{\lambda(\theta)}{1+\lambda(\theta)} d \theta=-\infty
$$

Also, if $F$ is bounded then (6) is equivalent to

$$
\int_{0}^{2 \pi} \log \lambda(\theta) d \theta=-\infty
$$

Proof. We assume

$$
\int_{0}^{2 \pi} \log \frac{\lambda(\theta)}{1+\lambda(\theta)} d \theta>-\infty
$$

and show that $f \notin E s(F)$. Since $\lambda(\theta) /(1+\lambda(\theta))<1$ our assumption implies by $\log (\lambda(\theta) /(1+\lambda(\theta)))$ is integrable on $[0,2 \pi]$. Let $d(z ; D)$ denote the distance between $z$ and $D$. Since $D$ is a Jordan domain, there exists $\varepsilon, 0<\varepsilon<1$, such that $E=\{z: d(z, D) \leqslant \varepsilon\}$ avoids an open set. The function $\log \frac{\varepsilon}{2}(\lambda(\theta) /(1+\lambda(\theta)))$ is also integrable on $[0,2 \pi]$. Let

$$
g(z)=z \exp \left[\frac{1}{2 \pi} \int_{0}^{2 \pi} \frac{e^{i t}+z}{e^{i t}-z} \log \frac{\varepsilon}{2} \frac{\lambda(t)}{1+\lambda(t)} d t\right] .
$$


(8) gives $|g(z)|<\varepsilon / 2$ for $z \in \Delta$ and it is known that

$$
\left|g\left(e^{i \theta}\right)\right|=\frac{\varepsilon}{2} \frac{\lambda(\theta)}{1+\lambda(\theta)}
$$

for almost all $\theta$. Since $\lambda(\theta)$ is the distance between $f\left(e^{i \theta}\right)$ and $\partial D$ and $\left|g\left(e^{i \theta}\right)\right|<$ $\lambda(\theta) / 2$, it follows that $f\left(e^{i \theta}\right) \pm g\left(e^{i \theta}\right) \in \bar{D}$ for almost all $\theta$. Note that $g \neq 0$. If $h=f \pm g$ then $\left|g\left(e^{i \theta}\right)\right|<\varepsilon / 2$ implies $h(\Delta) \subset E$. Lemma 1 now implies $f(z) \pm g(z)$ $\in D$ for all $z \in \Delta$. Since $g(0)=0$ and $f \in s(F)$, we conclude $f \pm g \in s(F)$ and so $f \notin E s(F)$. This completes the proof.

Remark. Since $E H s(F) \subset E s(F),(6)$ holds if $f \in E H s(F)$.

THEOREM 3. Let $F \in \mathbb{Q}$ be univalent in $\Delta$. If $D=F(\Delta)$ is a Jordan domain then $E s(F) \subset\left\{F \circ w: w \in E B_{0}\right\}$.

Proof. Let $g=F \circ w \in E s(F)$ where $w \in B_{0}$. We shall prove that

$$
\int_{0}^{2 \pi} \log \left(1-\left|w\left(e^{i \theta}\right)\right|\right) d \theta=-\infty
$$

and so conclude that $w \in E B_{0}$. If we let $\lambda(\theta)$ be the distance between $g\left(e^{i \theta}\right)$ and $\partial D$, then Theorem 2 gives

$$
\int_{0}^{2 \pi} \log \frac{\lambda(\theta)}{1+\lambda(\theta)} d \theta=-\infty
$$

Let $E=\{\theta: \lambda(\theta)$ exist and $\lambda(\theta) \leqslant 1\}$ and let $G=\{\theta: \lambda(\theta)$ exist and $\lambda(\theta)>1\}$. Clearly $m(E \cup G)=2 \pi$. For $\theta \in E$, we have

$$
\frac{\lambda(\theta)}{2} \leqslant \frac{\lambda(\theta)}{1+\lambda(\theta)} \leqslant \lambda(\theta),
$$

and for $\theta \in G$, we have $1+\lambda(\theta)<2 \lambda(\theta)$ and so

$$
\frac{1}{2}<\frac{\lambda(\theta)}{1+\lambda(\theta)}<1
$$

Equation (10) implies

$$
\int_{G} \log \frac{\lambda(\theta)}{1+\lambda(\theta)} d \theta
$$

is convergent. Therefore,

$$
\int_{0}^{2 \pi} \log \frac{\lambda(\theta)}{1+\lambda(\theta)} d \theta=\int_{E} \log \frac{\lambda(\theta)}{1+\lambda(\theta)} d \theta=-\infty
$$

and by (9) $\int_{E} \log \lambda(\theta) d \theta=-\infty$. Now

$$
\int_{0}^{2 \pi} \log \lambda(\theta) d \theta=\int_{G} \log \lambda(\theta) d \theta+\int_{E} \log \lambda(\theta) d \theta .
$$


By Theorem $1, \int_{0}^{2 \pi} \log ^{+} \lambda(\theta) d \theta$ is convergent and so $\int_{G} \log \lambda(\theta) d \theta=$ $\int_{G} \log ^{+} \lambda(\theta) d \theta$ is convergent. Since $\int_{E} \log \lambda(\theta) d \theta=-\infty$ we conclude from (11) that $\int_{0}^{2 \pi} \log \lambda(\theta) d \theta=-\infty$. Again, by Theorem 1, we conclude that

$$
\int_{0}^{2 \pi} \log \left(1-\left|w\left(e^{i \theta}\right)\right|\right) d \theta=-\infty
$$

Hence $w \in E B_{0}$.

ReMARK. We note that $E H s(F) \subset\left\{F \circ w: w \in E B_{0}\right\}$ since $E H s(F) \subset E s(F)$.

\section{REFERENCES}

1. Y. Abu-Muhanna, On extreme points of subordination families, Proc. Amer. Math. Soc. 87 (1983), 439-443.

2. P. L. Duren, Theory of $H^{p}$ spaces, Academic Press, New York, 1970.

3. Chr. Pommerenke, Univalent functions, Vandenhoeck and Ruprecht, Göttingen, 1975.

Department of Mathematical Sciences, University of Delaware, Newark, Delaware 19711 